\title{
Biomechanical and Histological Assessment of a Polyethylene Terephthalate Screw Retention Technology in an Ovine Metatarsal Fracture Model
} \author{
Kirk C. McGilvray2 ${ }^{2}$ \\ ${ }^{1}$ Department of Clinical Sciences, Preclinical Surgical Research \\ Laboratory (PSRL), Colorado State University, Fort Collins, Colorado, \\ United States \\ 2 Department of Mechanical Engineering, Orthopaedic \\ Bioengineering Research Laboratory (OBRL), Colorado State \\ University, Fort Collins, Colorado, United States \\ ${ }^{3}$ Savannah, Georgia, United States \\ Vet Comp Orthop Traumatol 2020;33:153-160.
}

Jeremiah Easley ${ }^{1}$ Christian Puttlitz ${ }^{2}$ Cecily Broomfield ${ }^{2}$ Ross Palmer ${ }^{1}$ Alexander Jones ${ }^{3}$

\begin{abstract}
Address for correspondence Kirk C. McGilvray, PhD, 1374 Campus Delivery, Fort Collins, CO 80523-1374, United States (e-mail: kirk. mcgilvray@colostate.edu).
\end{abstract}

\begin{abstract}
Keywords

- screw loosening

- screw failure

- fracture healing

- ovine

- screw retention
\end{abstract}

Objective Screw loosening in fracture fixation poses a clinical risk which may lead to implant failure, particularly in poor bone quality. The objective of this study was to examine the effectiveness of a novel screw retention technology (SRT) for increased screw purchase in a large animal metatarsal fracture model.

Study Design This was a biomechanical, radiographic, and histological study utilizing an ovine metatarsal fracture model. Twenty-four sheep metatarsi underwent 3-mm ostectomies and were repaired with a nine-hole plate and 3.5-mm screws placed in oversized 3.5-mm holes to simulate worst case revision surgeries (i.e. no initial screw thread bone contact). Sheep were sacrificed at 3, 6 or 12 weeks ( $n=6$ each) postoperation. Post-sacrifice, each surgically implanted screw underwent either destructive mechanical testing or histomorphometric analyses.

Results Treated metatarsi showed improved screw retention and normal fracture healing. Significant improvement in breakout strength and pullout strength of screws treated with the SRT were found as a function of healing time. Histologically, bone ingrowth at the screw interface was also shown to significantly increase with healing time. Improvements in fracture healing, indicated by an increase in bone fraction and decrease in void space at the osteotomy, were also observed with healing time.

Conclusion The results demonstrate the effectiveness of the SRT as a method for improved screw retention in a rescue-screw type scenario.

\section{Introduction}

Screws are the most commonly used implant in orthopaedic surgery. ${ }^{1}$ However, screws do not always operate effectively and may loosen leading to fracture non-union. ${ }^{2,3}$ Screw failure is especially prevalent following procedures that treat patients with poor bone quality, ${ }^{3-5}$ and is one of the primary causes of revision procedures for orthopaedic hardware. ${ }^{4}$ These revision procedures typically require extensive preoperative planning, the use of specialized implants and tools and mastery of technically challenging surgical techniques that dramatically raise health care costs. ${ }^{6}$ There is a wide range of screw failure processes that can include screw backout, stripping, complete fracture or loosening due to received

May 3, 2019

accepted after revision

November 9, 2019

published online

February 23, 2020 (c) 2020 Georg Thieme Verlag KG Stuttgart · New York
DOI https://doi.org/ 10.1055/s-0039-3402518. ISSN 0932-0814. 
infection. ${ }^{3,7}$ Screw loosening is considered an unsolved issue and new techniques or devices that prevent loosening would represent a significant development for orthopaedic clinical practice.

To reduce the incidence of screw loosening, various groups have focused on improving screw/plate technologies, ${ }^{8-11}$ among other approaches including bone cements, materials from the operating room and high friction surface coatings. ${ }^{12-14}$ Many of these efforts are mechanical solutions implemented intraoperatively and carry additional sets of risks such as undue bone-implant pressure, compromised bone stability or increased bone removal. The most popular competing solutions are rescue screws (a screw with a larger diameter) ${ }^{11}$ and locking plate systems (systems that lock the screw and plate together). ${ }^{7,15}$ However, current solutions have not adequately addressed screw loosening by these hardware advancements. ${ }^{7,13,14}$

Accordingly, a novel rescue screw technology has been proposed that directly engineers the bone-screw interface (-Fig. 1). Succinctly, a unique bio-textile was fabricated into a braided sleeve; placed around the screw increasing the surface area of contact between the screw and the bone, thus enhancing screw engagement to prevent loosening. ${ }^{16}$ The screw retention technology (SRT) studied here uses a cylindrical braided device composed of polyethylene terephthalate mono filaments; a member of the polyester family with no additives, it is not bioabsorbable. ${ }^{16}$ Polyethylene terephthalate has previously demonstrated biocompatibility in many clinical applications, including cardiovascular grafts, ${ }^{17}$ plastic surgery application, ${ }^{18,19}$ artificial ligaments ${ }^{20,21}$ and bone augmentation. ${ }^{22}$

In this application, the bio-textile interface provides a compliant layer between the screw and bone; mechanical loads are distributed to reduce the pressure-induced bone resorption that frequently occurs at the screw-bone interface.

The objective of this study was to investigate the ex vivo biomechanics and histological composition of ovine metatarsal fracture model treated with a fixation plate and the
SRT. The post-implantation ex vivo breakout and pullout strength biomechanics were determined. In addition, the bone ingrowth adjacent to screws and callus healing was evaluated via histomorphometry.

\section{Materials and Methods}

This investigation was approved by the Institutional Animal Care and Use Committee (IACUC no. 16-6379). This study used 24 skeletally mature sheep that underwent a unilateral ostectomy and subsequent hardware implantation on their right metatarsus. Three time points at 3, 6 and 12 weeks postoperatively were used; six sheep were sacrificed at each of the three time points.

Sheep metatarsal bones underwent a $3 \mathrm{~mm}$ mid-diaphyseal transverse ostectomy that was stabilized with a 9-hole, $3.5 \mathrm{~mm}$ LC-DCP plate (DePuy Synthes; West Chester, Pennsylvania, United States) using seven proximally placed bicortical $3.5 \mathrm{~mm}$ cortical screws (DePuy Synthes; West Chester, Pennsylvania, United States) and two distally placed unicortical $4.0 \mathrm{~mm}$ cancellous screws (DePuy Synthes). To simulate a scenario requiring rescue screws, $3.5 \mathrm{~mm}$ pilot holes were drilled for the seven proximal bicortical screws and $4.0 \mathrm{~mm}$ pilot holes were created for each of the distal screws. This surgical model was utilized to induce the worst possible case of screw-bone engagement (e.g. no screwbone engagement without SRT augmentation). The SRT device (OGmend Implant System; Woven Orthopedic Technologies, LLC Manchester, Connecticut, United States) was slid over the outer diameter of all screws. ${ }^{16}$ The length of the SRT was matched to the length of the screw body and placed into the pilot hole using a stylus, leaving $\leq 1 \mathrm{~mm}$ portion of the device exposed. The exposed portion of the SRT implant was monitored to ensure the device did not migrate.

Terminal in vivo insertion torque $(\mathrm{N}-\mathrm{m})$ was measured during surgery using a torque sensing screwdriver (TAT300; Futek, Inc., Irvine, California, United States). Following healing,

\section{Screw Retention Technology (SRT)}

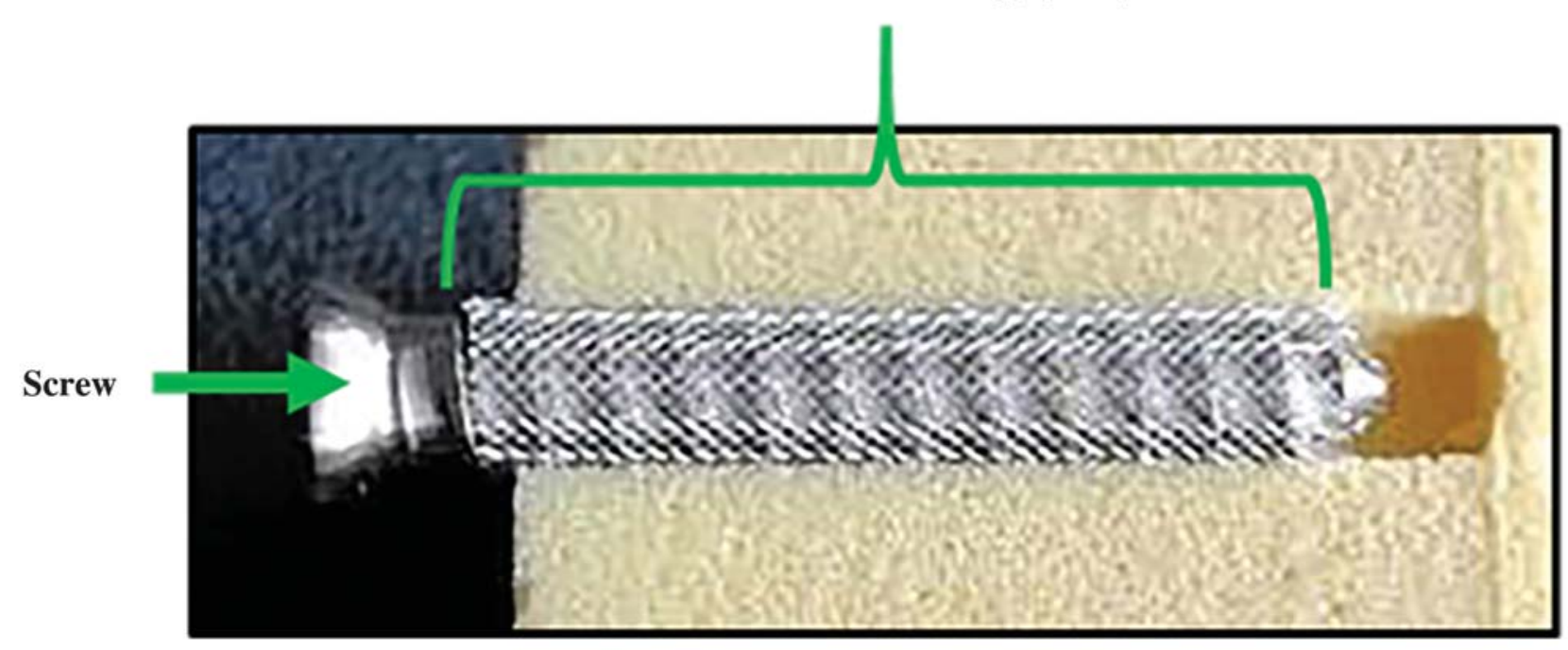

Fig. 1 Example image of a novel rescue screw retention technology designed to directly engineer the bone-screw interface. The implant comprises a unique bio-textile (i.e. a braided sleeve is placed around the screw). 

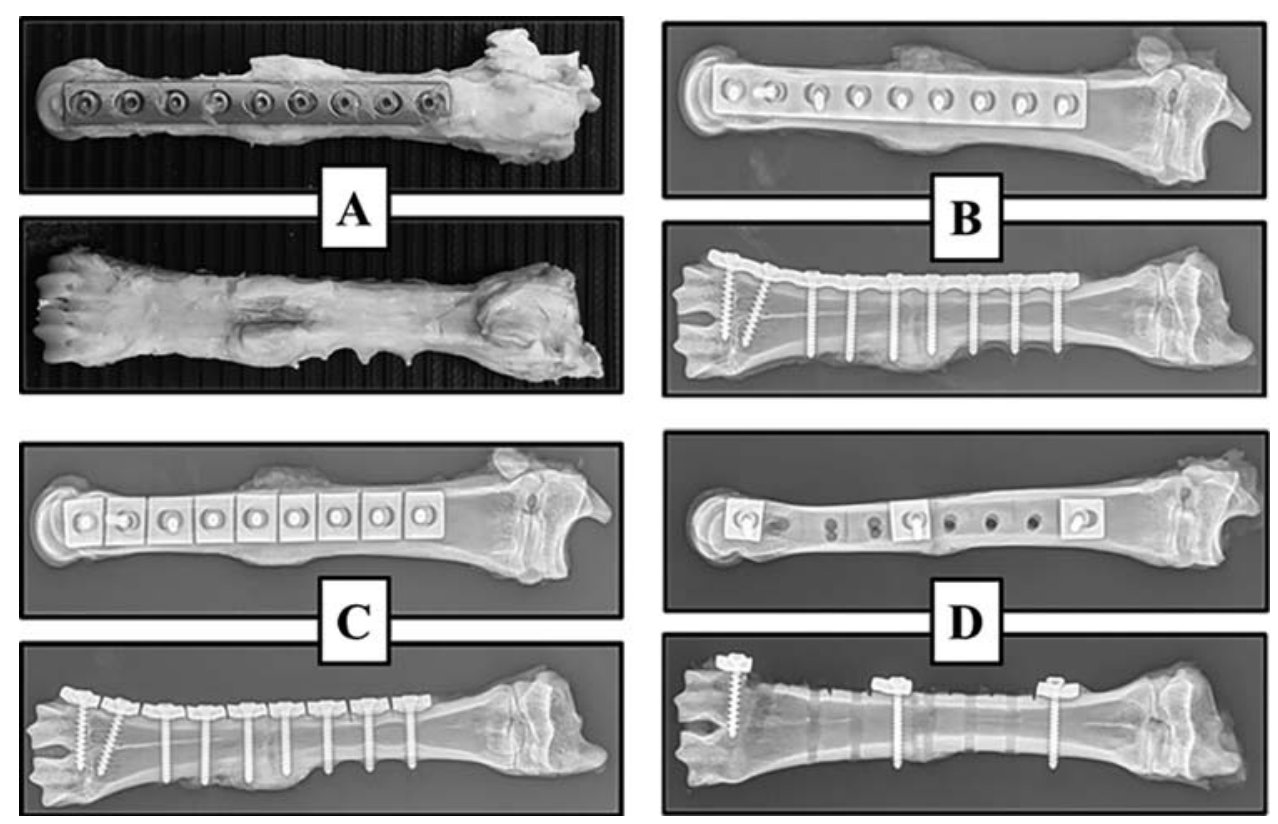

Fig. 2 Example images of metatarsals in the study, including (A) radiograph at time 0, (B) photograph following dissection, (C) radiograph prior to screw pullout showing cut plate and (D) radiograph following screw pullout showing remaining screws for histology.

euthanasia and gross dissection, freshly harvested metatarsal samples were prepared; extraneous soft-tissue was removed, taking great care not to damage the screw insertion, SRT implant, callus or ostectomy.

Following gross dissection, the bone samples were photographed and subject to biplanar digital radiography (-Fig. 2A and 2B). Screw breakout torque (defined as the initial break-out moment [ $\mathrm{N}-\mathrm{mm}]$ required to loosen the screw) was also determined for $n=3$ of 9 screws per sample by the digital torque sensing screwdriver.

Destructive screw pullout force was also determined for $n=3$ of 9 screws per sample. Fracture fixation plates were cut using a rotary cutoff wheel to isolate each screw (-Fig. 2C). Care was taken to ensure that the isolation process did not detrimentally affect the adjacent screws; samples were irrigated with saline to minimize thermal effects. Metatarsi were then rigidly mounted into a testing system (Mini Bionix 858, MTS System, Eden Prairie, Minnesota, United States). The long axis of the screw was aligned collinearly with the actuator to ensure a normal vector pullout direction. Individual screw-plate constructs were quasi-statically withdrawn at a rate of $1 \mathrm{~mm} / \mathrm{s}$. Force $(\mathrm{N})$ and displacement $(\mathrm{mm})$ data were collected at $150 \mathrm{~Hz}$. Construct stiffness $(\mathrm{N} / \mathrm{mm})$ and ultimate failure load $(\mathrm{N})$ were calculated for samples allocated to pullout testing. A total of 18 screws were investigated each for breakout and pullout at each time point.

Histological analyses were conducted on the ostectomy fracture to demonstrate the quality of healing at the bone defect site, contributing six tissue samples per time point. Osteotomy site samples were processed using standard decalcified paraffin techniques and stained with haematoxylin and eosin.

The remaining three screws in each metatarsal (-Fig. 2D) were used for non-decalcified hard tissue histology, yielding a total of 18 screws for histological evaluation at each time point. Samples were processed using standard non-decal- cified techniques. ${ }^{16,23,24}$ Sections were taken along the long axis of the screw to display the implant and surrounding bone. Sections were stained with Sanderson's Rapid Bone, and then counterstained using Van Gieson's solution.

Images were acquired for the entire section using a microscope (AG Heinze; Lake Forest, California, United States) and digital camera (Diagnostic Instruments; Sterling, Heights, Michigan, United States). Image Pro software (Media Cybernetics, Silver Spring, Maryland, United States) was used for histomorphometric measurements.

The ostectomy region of interest (ROI) was set as the area from the proximal surgical osteotomy cut to the distal cut. The screw ROI was set as the length of screw within the bone with an area extending $300 \mu \mathrm{m}$ towards the centreline of the screw and $300 \mu \mathrm{m}$ into the native bone. The histomorphometric parameters measured within each ROI were percent bone area within the ROI (\%), percent fibrous/soft tissue within the ROI (\%), percent implant (as applicable for screw and SRT device) area within the ROI (\%) and percent void area within the ROI (\%).

Histology sections were also evaluated by a certified pathologist to document the cellular responses for each of the samples. The pathologist was blinded to the treatment group. The sections were qualitatively analysed according to cell type (i.e. polymorphonuclear, lymphocytes, plasma, macrophages, giant and osteoblastic cells) and implant responses (i.e. signs of bone remodelling, implant degradation and neovascularization).

Significance was determined using a standard one-way analysis of variance (ANOVA) test, where $p$-values less than 0.05 were considered to be significant (SigmaStat; Systat Software Inc., San Jose, California, United States). A post-hoc Student-Newman-Keuls multiple comparison analysis was performed to determine statistically relevant $p$-values. Significant differences are designated with similar letters. The statistical power for any comparison was above 0.80 . 


\section{Results}

Clinical assessment, as observed by two board certified veterinary surgeons (J.T.E and R.H.P.), of screw insertion in the oversized holes indicated that surgical screw insertion with SRT augmentation felt surgically tight and clinically acceptable during in vivo implantation. All animals survived to term and gross necropsy yielded no adverse findings. Postsacrifice radiographs indicated normal osteotomy healing, no screw backout or plate migration. All biomechanical and histological tests were run to completion and no experimental issues were noted.

For cortical screw trajectories, the terminal insertion torque (mean \pm one standard deviation) with the SRT augmentation was $0.34 \pm 0.10 \mathrm{~N}-\mathrm{m}, 0.43 \pm 0.15 \mathrm{~N}-\mathrm{m}$ and $0.37 \pm 0.12 \mathrm{~N}$-m for 3, 6 and 12-week groups respectively. Similarly, the terminal insertion torque for cancellous screws was $0.45 \pm 0.20 \mathrm{~N}-\mathrm{m}, 0.61 \pm 0.27 \mathrm{~N}-\mathrm{m}$ and $0.36 \pm 0.17 \mathrm{~N}-\mathrm{m}$ for 3, 6 and 12-week groups respectively. No significant difference in insertion torque was found between groups for either the cancellous or cortical screws.

Three-, 6- and 12-week group cortical screw breakouts exhibited mean torques of $0.07 \pm 0.03 \mathrm{~N}-\mathrm{m}, 0.14 \pm 0.08 \mathrm{~N}-\mathrm{m}$ and $0.15 \pm 0.09 \mathrm{~N}-\mathrm{m}$, respectively. Despite no significant difference between the 6- and 12-week groups, both 6and 12-week groups had significantly larger breakout torque magnitudes as compared with the 3-week group $(p<0.01)$.

Screw pullout force $(\mathrm{N})$ and stiffness $(\mathrm{N} / \mathrm{mm})$ data are presented (-Fig. 3). The mode of failure was consistent across sacrifice time points with screws failing under straight axial displacement with mild/moderate bone avulsion. No micro-motion at the screw-plate interface was observed.

Cortical and cancellous pullout forces for screws with cortical trajectories are shown ( - Fig. $\mathbf{3 A}$ and $\mathbf{3 B}$, respectively). Cortical screw pullout forces were significantly different, with pullout forces significantly increasing between all three sacrifice time points (all $p<0.01$ ). Cancellous pullout forces also demonstrated significant increases in magnitude as a function of healing time $(p=0.02)$, with the lone exception that there was not a significant increase between the 6- and 12-week time points $(p=0.06)$.

The results of histomorphometric analyses for screw ROI and ostectomy ROI are shown in -Tables $\mathbf{1}$ and 2, respectively). No statistical differences were calculated for total ROI areas across sacrifice time points ( $p$-values of $0.93,0.84$ and 0.73 for the cortical screw, cancellous screw and osteotomy ROI respectively).
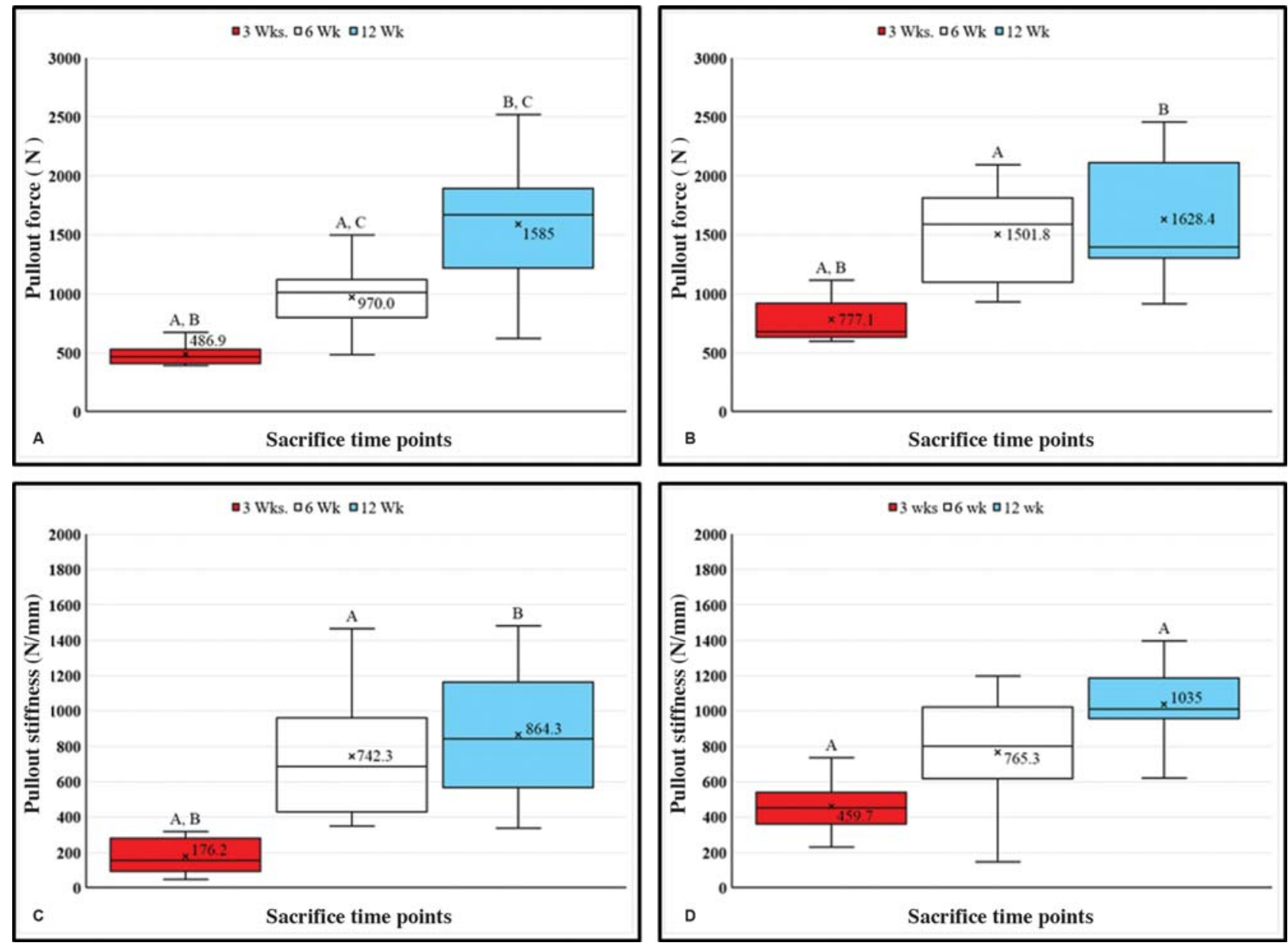

Fig. 3 Screw pullout data of metatarsals treated with screw retention technology after 3,6 and 12 weeks, showing (A) cortical screw pullout force (A, B, C: $p<0.01$ ), (B) cancellous screw pullout force (A, B: $p=0.02)$, (C) cortical screw pullout stiffness (A, B: $p<0.01)$, and (D) cancellous screw pullout stiffness $(A: p=0.01)$. 
Table 1 Histomorphometric data (mean \pm standard deviation) for cortical and cancellous screw ROI

\begin{tabular}{|l|l|l|l|l|l|l|}
\hline \multirow{2}{*}{$\begin{array}{l}\text { Constituents of } \\
\text { interest }\end{array}$} & \multicolumn{4}{|l|}{ Cortical screws } & \multicolumn{3}{l|}{ Cancellous screws } \\
\cline { 2 - 7 } & $\mathbf{3} \mathbf{~ W k}$ & $\mathbf{6} \mathbf{~ W k}$ & $\mathbf{1 2} \mathbf{~ W k}$ & $\mathbf{3} \mathbf{~ W k}$ & $\mathbf{6} \mathbf{~ W k}$ & $\mathbf{1 2} \mathbf{~ W k}$ \\
\hline \% Bone & $16.60 \pm 3.70^{\mathrm{A}}$ & $17.90 \pm 4.90^{\mathrm{B}}$ & $21.5 \pm 5.40^{\mathrm{A}, \mathrm{B}}$ & $10.10 \pm 4.30^{\mathrm{C}}$ & $14.80 \pm 5.30^{\mathrm{D}}$ & $17.90 \pm 6.60^{\mathrm{C}, \mathrm{D}}$ \\
\hline \% Soft tissue & $8.06 \pm 4.13^{\mathrm{E}}$ & $8.88 \pm 6.40^{\mathrm{F}}$ & $5.42 \pm 3.32^{\mathrm{E}, \mathrm{F}}$ & $12.8 \pm 4.70^{\mathrm{G}, \mathrm{H}}$ & $6.42 \pm 2.80^{\mathrm{G}}$ & $7.74 \pm 4.67^{\mathrm{H}}$ \\
\hline \% Implant & $3.35 \pm 1.73$ & $2.99 \pm 1.53$ & $3.04 \pm 1.95$ & $2.74 \pm 1.14$ & $2.18 \pm 1.10$ & $3.07 \pm 1.35$ \\
\hline \% Screw & $57.6 \pm 7.20$ & $53.7 \pm 11.40$ & $58.90 \pm 5.90$ & $47.10 \pm 7.60$ & $42.10 \pm 14.90$ & $43.40 \pm 9.50$ \\
\hline \% Void space & $14.40 \pm 5.60^{\mathrm{I}}$ & $16.60 \pm 6.90^{\mathrm{J}}$ & $11.20 \pm 4.50^{\mathrm{l}, \mathrm{J}}$ & $27.20 \pm 5.50$ & $34.50 \pm 17.70$ & $27.80 \pm 9.60$ \\
\hline
\end{tabular}

Abbreviation: ROI, region of interest.

Note: Significant differences are indicated by like letters (A, B, D, E, G, H, I, J: $p<0.01 ; \mathrm{C}: p<0.04 ; \mathrm{F}: p<0.03$ ).

Table 2 Histomorphometric data for osteotomy ROI

\begin{tabular}{|l|l|l|l|}
\hline $\begin{array}{l}\text { Constituents } \\
\text { of interest }\end{array}$ & $\mathbf{3} \mathbf{W k}$ & $\mathbf{6} \mathbf{W k}$ & $\mathbf{1 2} \mathbf{~ W k}$ \\
\hline \% Bone & $43.20 \pm 12.80$ & $46.70 \pm 14.80$ & $53.40 \pm 9.70$ \\
\hline \% Soft tissue & $13.00 \pm 8.00$ & $16.20 \pm 12.80$ & $14.00 \pm 9.40$ \\
\hline \% Void space & $43.70 \pm 10.70$ & $38.40 \pm 14.30$ & $32.60 \pm 10.90$ \\
\hline
\end{tabular}

Abbreviation: ROI, region of interest.

Note: No significant differences were found.
Histopathology showed that the SRT sleeves were embedded within reactive fibrosis and associated with a rare population of lymphocytes and few macrophages. The SRT sleeve was observed to be embedded within the bone in most cases ( - Fig. 4). Qualitative histopathology analysis indicated that there were no signs of abnormal gross cellular reactions (inflammation or infection) at the bone-screw-SRT interfaces or osteotomy sites. There were also no gross signs of device degradation or debris indicating the device maintained its structural integrity throughout the study.

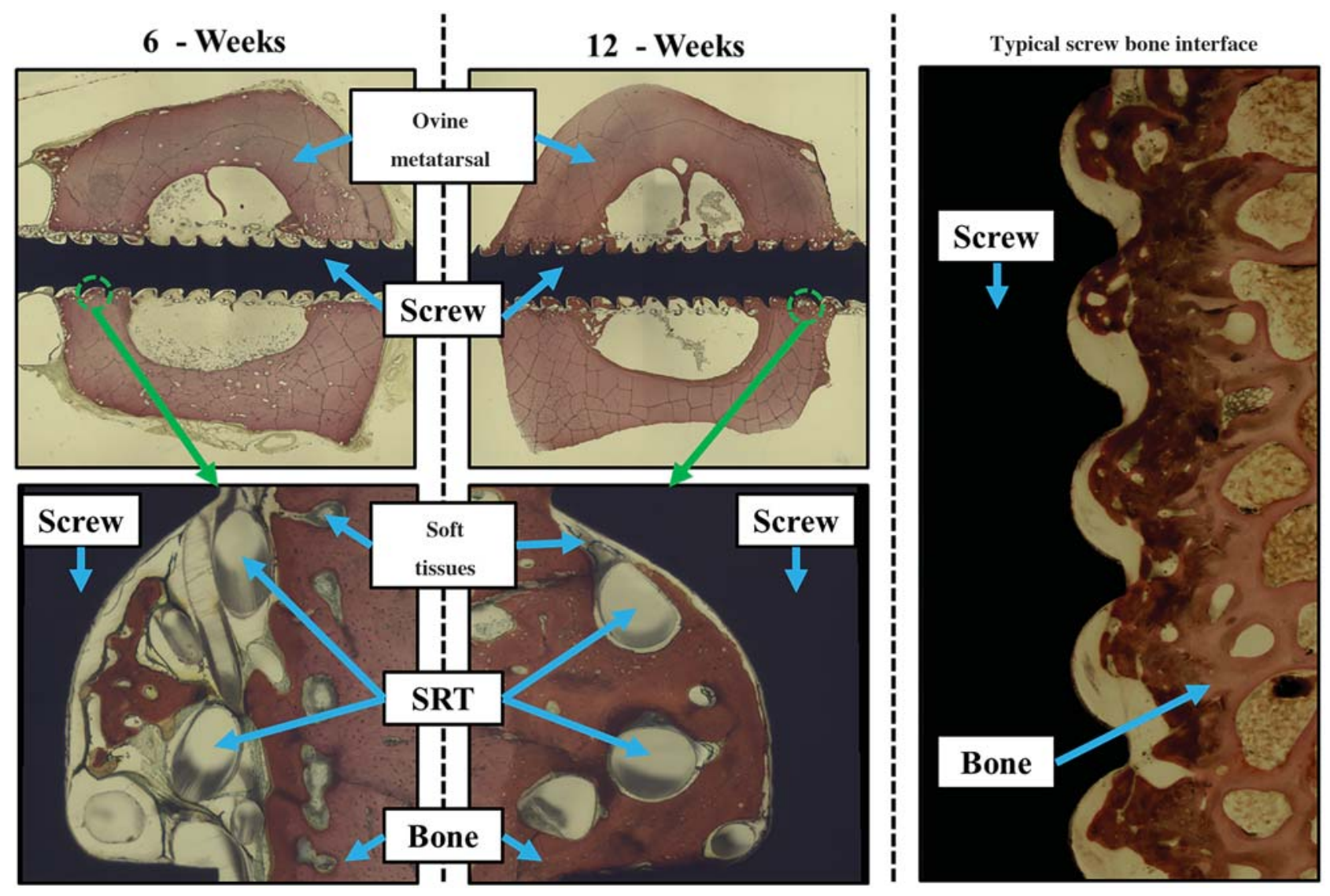

Fig. 4 (Top left and top middle) Example histologic images demonstrating the histomorphometric region of interest (i.e. the screw-bone interface) at $10 x$ magnification at 6 and 12 weeks post-implantation. (Bottom left and bottom middle) Example images highlighting the implant, screw retention technology (SRT) device, bone and soft tissue at the screw-bone interface at 100x magnification at 6 and 12 weeks postimplantation. (Right) Example image (derived from an unrelated study; unpublished data) showing a typical control screw-bone interface in the ovine metatarsal at 12 weeks post-implantation. 


\section{Discussion}

Pullout and breakout biomechanical testing exhibited consistent improvements in screw retention as a function of increased healing times. Histologically, increased bone fraction surrounding the cortical and cancellous screws consistent with improved screw retention was observed following healing. Increased bone fraction and decreased void fraction, as well as radiographic changes, were identified at the ostectomy indicating successful progression of osteotomy healing toward osseous union consistent with metatarsal osteotomy healing previously observed by our group utilizing standard screw-bone engagement models. ${ }^{25-28}$

Unfortunately, no previous studies in the literature were found on screw pullout and breakout in ovine metatarsals for direct comparison. However, sheep metatarsi are commonly used as an analogue for human tibia due to similarities in size and bone mineral density between the two bones. ${ }^{25-27}$

Matityahu and colleagues ${ }^{29}$ evaluated the pullout strength of 3.5-mm self-tapping screws in a standard drill hole model using cadaveric diaphyseal tibiae. It was found that a single insertion, four insertions and five insertions yielded pullout strengths of $1710 \pm 550 \mathrm{~N}, 1030 \pm 543 \mathrm{~N}$ and $364 \pm 209 \mathrm{~N}$ respectively. These findings are of a similar magnitude to the pullout strengths of the present study and indicate that, after 12 weeks of healing, the SRT provides similar screw retention to screws with one insertion and no over drilling. In addition, our data indicated that after 3 weeks of healing, the SRT provides greater screw retention than screws with five insertions and no overdrilling. Similarly, Oldakowska and colleagues ${ }^{30}$ evaluated the pullout strength of $4 \mathrm{~mm}$ self-tapping screws in a standard drill hole model in cadaveric thoracic ovine bone. It was found that the screws exhibited a failure force of $695.0 \pm 82.4 \mathrm{~N}$, and a stiffness of $618.5 \pm 114.1 \mathrm{~N} / \mathrm{mm}$. Again, these findings are of a similar magnitude to the pullout and stiffness data generated for this study, which utilized an overdrilled hole model, following 6 and 12 weeks of healing. The similarity between our data and these studies indicates that the SRT may allow relevant healing at the screw-bone interface in an overdrilled (i.e. rescue) screw scenario following healing.

Claes and colleagues ${ }^{31}$ explored the tissue differentiation of ovine metatarsal ostectomies $(2.1 \mathrm{~mm})$ following 9 weeks of healing subject to the established method of external fixation. Despite discrepancies in healing times and fracture size, all values in the present study were within one standard deviation of all reported cortical and medullary tissue distributions for bone, soft tissue and connective tissue/void space. Indeed, even the 3-week time point in the present study observed similar ostectomy tissue differentiation to a smaller fracture after a longer healing duration. Similarly, Augat and colleagues ${ }^{32}$ also investigated external fixation of $2.0 \mathrm{~mm}$ ovine metatarsal ostectomies. Following 9 weeks of healing, histological data at the ostectomy yielded similar composition of bone, soft tissue and connective tissue/void space to the present study. Histomorphometric data indicated the percent bone increased, the percent soft tissue did not increase and the percent implant remained constant; these trends likely resulted from normal bone remodelling, lack of excessive fibrotic reaction to the SRT and no degradation of the SRT. This increase in bone likely resulted in the observed increases in mechanical integrity. The congruence of the tissue differentiation with the discussed studies indicates that effective fracture healing was induced in the treated group of this study. Therefore, it appears that the SRT was essential at promoting the appropriate mechanical integrity to allow for fracture healing.

When complications occur, either intraoperatively or in revision procedures, surgeons must remove additional bone stock to replace the loose screws, thus limiting the ability of the surgeon to generate the necessary stability and reduction for fracture fixation. When orthopaedic screws require revision, surgeons use a variety of ad-hoc techniques including the use of larger and/or longer screws (i.e. rescue screw), inserting screws in a different trajectory/pilot hole, use of additional plates or augmenting the failed hole with bone void fillers or polymethyl methacrylate (i.e. bone cements) or with the Matchstick method. ${ }^{33}$ Unfortunately, the use of a strip ('matchstick') of bone graft to act as a shim leads to asymmetric hoop stresses with force concentration at the strip of bone graft and increases the risk of screw hole wall fracture particularly with compromised (osteoporotic) bone quality. Cements also have several drawbacks. A major problem is the difficulty of precise placement and the prevention of inadvertent migration of the semi-liquid cement which could cause problems (i.e. mechanical impingement). In addition, the most commonly used orthopaedic cement, polymethyl methacrylate, in the process of in situ polymerization can cause local or systemic toxicity as in the case of monomer release. Also, there is the generation of high local temperatures as the cement exothermically polymerizes, with the potential for thermal injury to local structures. The main problem with repositioning approaches is the creation of stress risers with the empty screw holes weakening the underlying osseous structure; local anatomic considerations may also prevent plate repositioning. Therefore, there still exists a clinical need for a device which can prevent screw complications and revisions, and, if revision is required, provides a more robust stabilization augmentation in clinical surgery. The SRT device studied here does not suffer from these issues. The structure of the device maximizes screw bone engagement in a uniform circumferential fashion avoiding stress concentration. The SRT has no issues with either exothermic curing, or local toxicity as the material of the SRT has well-documented history of bio-compatibility. With the SRT, there is no need to leave a screw hole empty eliminating subsequent stress riser creation and obviating the need to reposition an orthopaedic plate or device.

The design of this study excluded an in vivo negative control group (i.e. overdrilled pilot hole with a standard screw). Subjectively, all screws in a negative control group were considered clinically unacceptable and unsafe for fracture repair in an in vivo setting by two board-certified veterinary surgeons. This was further validated by time-zero ramp to failure testing on cadaveric ovine metatarsal samples (data not shown); the measured failure load for a proposed negative control group was determined to be less than the estimated load on the treated limb at any time during in vivo healing. Accordingly, application of a negative control group in an in 
vivo animal model would have been ill-advised, in-humane and against the spirit of IACUC guidelines. It was determined that any negative control group samples would suffer catastrophic failure upon standing and full-weight-bearing immediately following recovery from surgery. A positive control (i.e. a screw placed in the surgically standard hole or the use of some form of rescue screw) was deemed to be the most appropriate control for this model. However, as this study was an initial attempt to prove the efficacy of the SRT device a positive control arm of the study was not implemented. By comparing our results to data from previous studies, ${ }^{29,30}$ that used the current standards of screw insertion, it appears that augmentation of an overdrilled hole with the SRT leads to similar levels of acute biomechanical stability following healing. This assertion is further strengthened by the fact that all fractures generated within this study had typical healing pathways leading towards clinically acceptable osseous union, which would be unlikely if the implanted hardware was not adequality stabilizing the fracture. A recent study examining the effectiveness of the SRT in an ovine spine model, in which a positive and negative control where possible, demonstrated that the SRT device does improve screw-bone purchase as compared with a negative control. ${ }^{16}$ However, while the literature is replete with studies that have translated the general results of sheep orthopaedic models to human applications, ${ }^{26,27,34,35}$ one should take caution when prescribing the absolute values of ovine-derived data to that of the human condition.

In conclusion, the novel SRT investigated in this studied showed improved screw retention in an in vivo ovine metatarsal model with oversized holes as healing time progressed, and that the biomechanical stability imparted by the SRT device was of the same order observed for standard screws implanted acutely. The biomechanical and histological results of this study demonstrate the SRT as an effective method for improved screw retention for mitigation of clinical screw failure in situations that might otherwise have clinically unsatisfactory fixation.

\section{Author's Contributions}

All authors drafted, revised and approved the submitted manuscript. Jeremiah Easley, Christian Puttlitz, Cecily Broomfield, Ross Palmer and Kirk C. McGilvray contributed to conception of study, study design, acquisition of data and data analysis and interpretation. Alexander Jones contributed to conception of study, study design, and data analysis and interpretation.

\section{Funding}

This work was funded by a research grant to Colorado State University (Fort Collins, Colorado, United States) from Woven Orthopedic Technologies (Manchester, Connecticut, United States).

\section{Conflict of Interest}

Dr Broomfield reports grants from Woven Orthopedic Technologies, Manchester, Connecticut, United States, during the conduct of the study. Dr Easley reports grants from Woven Orthopedic Technologies, Manchester, Connecticut, United States. Dr. Jones has a patent OGmend Implant System, Woven Orthopedic Technologies, LLC Manchester, Connecticut, United States. issued. Dr McGilvray reports grants from Woven Orthopedic Technologies, Manchester, Connecticut, United States, during the conduct of the study. Dr Palmer reports grants from Woven Orthopedic Technologies, Manchester, Connecticut, United States, during the conduct of the study. Dr Puttlitz reports grants from Woven Orthopedic Technologies, Manchester, Connecticut, United States, during the conduct of the study. The authors, Dr McGilvray, Dr Easley, Dr Puttlitz, Mrs Broomfield and Dr Palmer report no perceived or potential conflicts of interest that may have biased the work presented within this manuscript. Dr Jones holds the patent on the device described within this study.

\section{References}

1 Collinge C, Hartigan B, Lautenschlager EP. Effects of surgical errors on small fragment screw fixation. J Orthop Trauma 2006;20(06): 410-413

2 Galbusera F, Volkheimer D, Reitmaier S, Berger-Roscher N, Kienle A, Wilke HJ. Pedicle screw loosening: a clinically relevant complication? Eur Spine J 2015;24(05):1005-1016

3 Fisher WD, Hamblen DL. Problems and pitfalls of compression fixation of long bone fractures: a review of results and complications. Injury 1978;10(02):99-107

4 El Saman A, Meier S, Sander A, Kelm A, Marzi I, Laurer H. Reduced loosening rate and loss of correction following posterior stabilization with or without PMMA augmentation of pedicle screws in vertebral fractures in the elderly. Eur J Trauma Emerg Surg 2013;39 (05):455-460

5 Halvorson TL, Kelley LA, Thomas KA, Whitecloud TS III, Cook SD. Effects of bone mineral density on pedicle screw fixation. Spine 1994;19(21):2415-2420

6 Hak DJ, McElvany M. Removal of broken hardware. J Am Acad Orthop Surg 2008;16(02):113-120

7 Nieto H, Baroan C. Limits of internal fixation in long-bone fracture. Orthop Traumatol Surg Res 2017;103(1S):S61-S66

8 Wu ZX, Gong FT, Liu L, et al. A comparative study on screw loosening in osteoporotic lumbar spine fusion between expandable and conventional pedicle screws. Arch Orthop Trauma Surg 2012;132(04):471-476

9 Paré PE, Chappuis JL, Rampersaud R, et al. Biomechanical evaluation of a novel fenestrated pedicle screw augmented with bone cement in osteoporotic spines. Spine 2011;36(18):E1210-E1214

10 Takigawa T, Tanaka M, Konishi H, et al. Comparative biomechanical analysis of an improved novel pedicle screw with sheath and bone cement. J Spinal Disord Tech 2007;20(06):462-467

11 Wall SJ, Soin SP, Knight TA, Mears SC, Belkoff SM. Mechanical evaluation of a 4-mm cancellous "rescue" screw in osteoporotic cortical bone: a cadaveric study. J Orthop Trauma 2010;24(06): 379-382

12 Yerby SA, Toh E, McLain RF. Revision of failed pedicle screws using hydroxyapatite cement. A biomechanical analysis. Spine 1998;23 (15):1657-1661

13 Pechon PH, Mears SC, Langdale ER, Belkoff SM. Salvaging the pullout strength of stripped screws in osteoporotic bone. Geriatr Orthop Surg Rehabil 2013;4(02):50-52

14 Bronsnick D, Harold RE, Youderian A, Solitro G, Amirouche F, Goldberg B. Can high-friction intraannular material increase screw pullout strength in osteoporotic bone? Clin Orthop Relat Res 2015;473(03):1150-1154 
15 Greiwe RM, Archdeacon MT. Locking plate technology: current concepts. J Knee Surg 2007;20(01):50-55

16 Easley J, Puttlitz CM, Seim H III, et al. Biomechanical and histologic assessment of a novel screw retention technology in an ovine lumbar fusion model. Spine J 2018;18(12):2302-2315

17 Lejay A, Colvard B, Magnus L, et al. Explanted vascular and endovascular graft analysis: where do we stand and what should we do? Eur J Vasc Endovasc Surg 2018;55(04):567-576

18 Fanous N, Tournas A, Côté V, et al. Soft and firm alloplastic implants in rhinoplasty: why, when and how to use them: a review of 311 cases. Aesthetic Plast Surg 2017;41(02):397-412

19 Patel K, Brandstetter K. Solid implants in facial plastic surgery: potential complications and how to prevent them. Facial Plast Surg 2016;32(05):520-531

20 Dai Z, Bao W, Li S, Li H, Jiang J, Chen S. Enhancement of Polyethylene Terephthalate Artificial Ligament Graft Osseointegration using a Periosteum Patch in a Goat Model. Int J Sports Med 2016;37(06):493-499

21 Li S, Ma K, Li H, Jiang J, Chen S. The effect of sodium hyaluronate on ligamentation and biomechanical property of tendon in repair of Achilles tendon defect with polyethylene terephthalate artificial ligament: a rabbit tendon repair model. BioMed Res Int 2016; 2016:8684231

22 Ono W, Maruyama K, Ogiso M, Mineno S, Izumi Y. Implant insertion into an augmented bone region using the canine mandible augmented by the "casing method". Anat Rec(Hoboken) 2018;301(05): 892-901

23 McGilvray KC, Easley J, Seim HB, et al. Bony ingrowth potential of 3D-printed porous titanium alloy: a direct comparison of interbody cage materials in an in vivo ovine lumbar fusion model. Spine J 2018;18(07):1250-1260

24 McGilvray KC, Waldorff EI, Easley J, et al. Evaluation of a polyetheretherketone (PEEK) titanium composite interbody spacer in an ovine lumbar interbody fusion model: biomechanical, microcomputed tomographic, and histologic analyses. Spine J 2017;17 (12):1907-1916
25 Gadomski BC, McGilvray KC, Easley JT, et al. An in vivo ovine model of bone tissue alterations in simulated microgravity conditions. J Biomech Eng 2014;136(02):021020

26 Gadomski BC, McGilvray KC, Easley JT, et al. An investigation of shock wave therapy and low-intensity pulsed ultrasound on fracture healing under reduced loading conditions in an ovine model. J Orthop Res 2018;36(03):921-929

27 Gadomski BC, McGilvray KC, Easley JT, Palmer RH, Santoni BG, Puttlitz CM. Partial gravity unloading inhibits bone healing responses in a large animal model. J Biomech 2014;47(12):2836-2842

28 McGilvray KC, Unal E, Troyer KL, et al. Implantable microelectromechanical sensors for diagnostic monitoring and post-surgical prediction of bone fracture healing. J Orthop Res 2015;33(10):1439-1446

29 Matityahu A, Hurschler C, Badenhop M, et al. Reduction of pullout strength caused by reinsertion of 3.5-mm cortical screws. J Orthop Trauma 2013;27(03):170-176

30 Oldakowski M, Oldakowska I, Kirk TB, et al. Pull-out strength comparison of a novel expanding fastener against an orthopaedic screw in an ovine vertebral body: an ex-vivo study. J Med Eng Technol 2016;40(02):43-51

31 Claes L, Eckert-Hübner K, Augat P. The fracture gap size influences the local vascularization and tissue differentiation in callus healing. Langenbecks Arch Surg 2003;388(05):316-322

32 Augat P, Margevicius K, Simon J, Wolf S, Suger G, Claes L. Local tissue properties in bone healing: influence of size and stability of the osteotomy gap. J Orthop Res 1998;16(04):475-481

33 Derinçek A, Türker M, Cinar M, Cetik O, Kalaycioğlu B. Revision of the failed pedicle screw in osteoporotic lumbar spine: biomechanical comparison of kyphoplasty versus transpedicular polymethylmethacrylate augmentation. Eklem Hastalik Cerrahisi 2012;23(02):106-110

34 Wilke HJ, Kettler A, Claes LE. Are sheep spines a valid biomechanical model for human spines? Spine 1997;22(20):2365-2374

35 Wilke HJ, Kettler A, Wenger KH, Claes LE. Anatomy of the sheep spine and its comparison to the human spine. Anat Rec 1997;247 (04):542-555 